\title{
A Decision Support Framework for Feasibility Analysis of International Space Station (ISS) Research Capability Enhancing Options
}

James N. Ortiz, Ph.D.

Mail Code AG

NASA, Johnson Space Center

2101 Nasa Parkway

Houston, Texas, 77058
Kelly Scott

Booz-Allen Hamilton

Worldwide Technology Business

2525 Bay Area Blvd, Suite 290

Houston, Texas, 77058

\section{Harold Smith \\ Raytheon Technical Services Co. Aerospace Engineering Services \\ 2224 Bay Area Blvd \\ Houston Texas, 77062}

\begin{abstract}
The assembly and operation of the ISS has generated significant challenges that have ultimately impacted resources available to the program's primary mission: research. To address this, program personnel routinely perform trade-off studies on alternative options to enhance research. The approach, content, level of analysis and resulting outputs of these studies vary due to many factors, however, complicating the Program Manager's job of selecting the best option. To address this, the program requested a framework be developed to evaluate multiple research-enhancing options in a thorough, disciplined and repeatable manner, and to identify the best option on the basis of cost, benefit, and risk. The resulting framework consisted of a systematic methodology and a decision-support toolset. The framework provides quantifiable and repeatable means for ranking research-enhancing options for the complex and multiple-constraint domain of the space research laboratory. This paper describes the development, verification and validation of this framework and provides observations on its operational use.
\end{abstract}




\section{Summary}

The assembly and operation of the International Space Station has generated significant unexpected challenges. These challenges have ultimately impacted resources available to the program's primary mission: research. To address these challenges, program personnel routinely perform technical trade-off studies on alternative options to enhance research. The approach, content, level of analysis and resulting outputs of these studies vary due to many factors, however, complicating the Program Manager's job of selecting the best option.

The program requested a methodology be developed to evaluate multiple researchenhancing options in a thorough, disciplined and repeatable manner, and to identify the best option on the basis of cost, benefit, and risk. To this end a NASA civilservice/contractor composite team was assembled, and the task was conducted in three phases.

During Phase I of the task, a new research capability enhancement decision-support paradigm comprising a systematic methodology and decision-support tool was created and demonstrated for the ISS Program Manager. In Phase II, the methodology and toolset were employed to identify and define options to enhance research onboard the ISS, and to evaluate the usefulness of the methodology and tools for future assessments by the ISS program. Finally, in Phase III, the toolset was refined to incorporate requested upgrades, and actual options under consideration by the program were analyzed to determine the most beneficial option on the basis of cost, benefit, and risk. The methodology and toolset were then handed off to ISS program personnel for future use.

At the heart of the task was a closed-loop methodology developed by the team. The objective of the methodology was to improve organizational focus and increase the emphasis of studies on potential on-orbit research productivity. The methodology comprised four major stages: strategic, tactical, implementation, and review. In the strategic and tactical stages, statistical analyses of representative time periods in the distant and near future were performed to assess potential benefits of research enhancement options. During the implementation phase, the program office performed the normal functions of defining and executing a project plan (including implementation of selected research enhancement options) that addressed requirements, budget, schedule, risk management, and an external review process. The review phase provided feedback to either validate, or drive adjustment to, the strategy. A database was created to house study results and to minimize duplication of study efforts. 
Supplementing the methodology was a purpose-built, spreadsheet-based tool. The Tool for Research Enhancement Decision Support (TREDS) was developed during Phase I and refined during the subsequent phases. The Analytical Hierarchy Process (AHP) documented in (Saaty 1983), was incorporated in TREDS to blend objective and subjective information toward summarizing the relative research merit of various options to the Program baseline. Objective information included three metrics chosen for their ability to represent research throughput, option costs, and option risks. Subjective information consisted of weightings the tool operator could apply to the three metrics to emphasize or de-emphasize their importance.

\section{Problem}

The International Space Station (ISS) now being assembled is designed to be a worldclass research facility. The ISS is the largest volume of long-duration, low-Earth-orbit, human-tended, multidisciplinary experimental environment, ever to be offered. Research opportunities contributing to the resolution of technological challenges on Earth and enabling humankind's departure from our solar system are becoming available as never before. Even during assembly, research operations are being conducted using steadily expanding research capabilities.

Assembling and putting into operation a spacecraft as complex as the ISS has generated significant unexpected program challenges. These challenges have ultimately impacted resources for the program's research mission. In the meantime, technical trade-off studies on alternative solutions that could mitigate this threat have continued; for example, the on-orbit crew time resource has been the subject of many such studies. The ISS Program Manager must evaluate the results of these alternative solution studies to select courses of action that are both technically and fiscally sound and defendable in the largest government policy framework. The fist step in this work was formulating a set of potential alternatives to enhance the research potential of the ISS. A summary of this work is shown in Figure 1. 


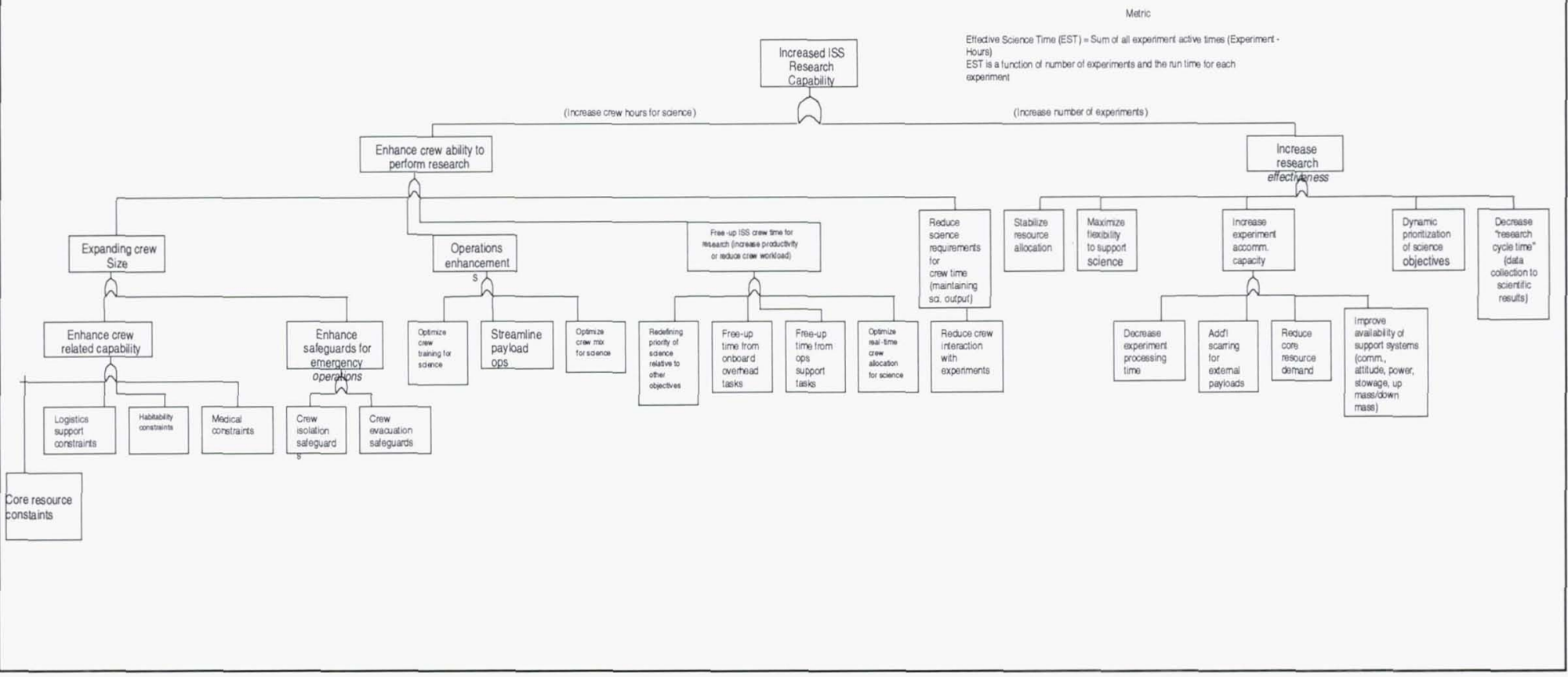

Figure 1. Research Enhancement Alternatives

The team then proceeded to demonstrate for the ISS Program Manager a new research capability enhancement decision-support framework comprising two components: a systematic, closed-loop methodology; and a complementary decision-support software tool.

\section{Methodology}

The objective of the proposed methodology was to improve organizational focus and increase studies emphasis on potential on-orbit research productivity. It comprised four major phases: strategy, tactical, implementation, and review. In the strategic phase, a statistical analysis of a representative ISS increment 4-6 years in the future would be performed to assess the research enhancement potential of alternatives during this time frame. The tactical phase would analyze specific increments 1-2 years in the future using actual payload planning data. During the implementation phase, the ISS Program Office (ISSPO) would perform its normal functions of defining and executing a project plan that addressed requirements, budget, schedule, risk management, and an external review process. It is during this phase that research enhancement options are implemented and realized research enhancement is measured. The review phase 
would provide the feedback that would either validate or adjust the strategy. This methodology is shown in Figure 2.

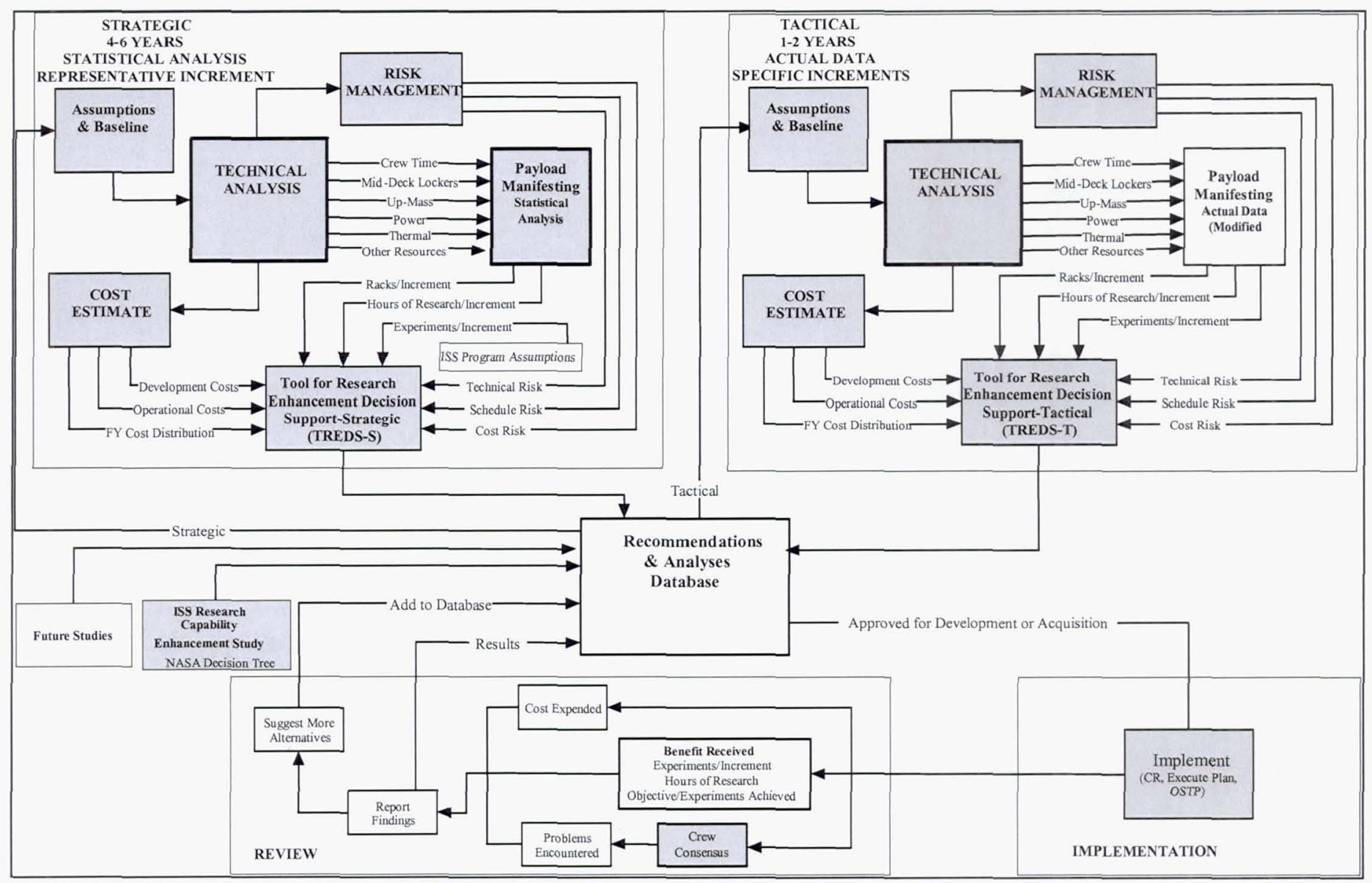

Figure 2. Research Enhancement Methodology

\section{Supporting Toolset}

The Tool for Research Enhancement Decision Support-Strategic or Tactical (TREDS-S or TREDS-T) was created to compare alternative options by calculating a research capability figure of merit (FOM) for each option. TREDS did this by using a modified form of the time-tested Analytical Hierarchy Process (AHP). AHP seemed appropriate because both objective and subjective inputs needed to be considered, and AHP had been developed specifically for this type of problem. The subjective value weightings of three benefits of an option (research diversity per research quarter; hours of research per research quarter; and number of research facilities operable per research quarter) and the quantities of these benefits per option, as forecast by the Program's statistical simulations model, were converted into normalized coefficients. The individual 
contributions of each option were then multiplied by these coefficients and summed to arrive at a single research return FOM for each option.

The research capability figure of merit is expressed mathematically by the following equation:

\section{Research Capability FOM =}

$\mathrm{C}_{\mathrm{RD}} * \mathrm{~W}_{\mathrm{RD}} *$ (Research Diversity) $+\mathrm{C}_{\mathrm{RH}} * \mathrm{~W}_{\mathrm{RH}} *$ (Hours of Research) $+\mathrm{C}_{\mathrm{RF}} * \mathrm{~W}_{\mathrm{RF}} *$ (Research Facilities Operable),

Where,

- $C_{X X}$ are the benefit coefficients relating the benefit metrics to the current baseline research capability

- $\mathrm{W}_{\mathrm{XX}}$ are the normalized weightings of preference for each metric, calculated by two matrix operations inherent to AHP $\left(0.09<=\mathrm{W}_{x x}<=0.81\right.$ and $\mathrm{W}_{\mathrm{RD}}+\mathrm{W}_{\mathrm{RH}}+$ $\left.\mathrm{W}_{\mathrm{RF}}=1.0\right)$.

The FOM was then used to calculate an Additional Research Multiple and a Cumulative Return, which were made the basis of TREDS standard output and defined as follows:

- The Additional Research Multiple was the FOM for a particular increment and represented the increase in research capability for an alternative. For example, a multiple of 1.2 indicated a research capability of 1.2 times the baseline, or a forecast increase in research capability of $20 \%$.

- Cumulative Return was the summation of the FOM over a given time period. For example, a cumulative research increase of $20 \%$ indicated that a particular alternative provided an increase in research capability of $20 \%$ over the baseline for the total time period under study. The baseline represents the research capability of the program if none of the research enhancement options are implemented.

The team also recommended the chartering of an "ISS Study Manager" to coordinate the methodology's technical, schedule, risk, cost, and AHP assessment components. Finally, the Phase I team recommended the development and initialization of a Recommendations \& Analyses Database. The R\&A Database would be under the Study Manager's direct control and serve as the central studies reference database for the ISS Program.

With these concepts preliminarily developed and demonstrated, the stage was set for their practical application. 


\section{Study Conduct}

The top-level approach to perform the studies is as follows: The study team would consider the various options appropriate to the study and advise the Study Manager, who would make the final selections. The Study Manager would then guide the team in obtaining the required data. Finally, the updated TREDS tool, in combination with the previously gathered data, would be used to rank-order the options based on their benefits, costs, and risks. The following paragraphs provide the main steps in the completions of a typical study

A) BASELINE DEFINITION. The main output of the TREDS tool is a representation of the increase in research return provided by each option as a percentage increase over baseline capability. To arrive at these figures, it was necessary to define the baseline research capability of the ISS for each time period under study. Efforts were directed toward identifying the baseline research capabilities provided by the ISS during the study timeframe. This required the definition of the ISS configuration during that timeframe to include the station elements, laboratory space and capabilities, key operations expected, allocations of crew time, frequency of resupply vehicles and up-mass capabilities.

B) SELECTION AND DEFINITION OF ALTERNATIVE OPTIONS. Options to be studied were drawn from those studied previously, and selected by the Study Manager on the basis of two criteria:

- Perceived level of benefit

- Availability and maturity of benefit information.

The total number of options to be studied in a typical study is around five. This number of options keeps the effort required at a reasonable level while still providing sufficient breadth of alternatives. The TREDS tool is capable of evaluating up to ten options at one time.

C) DEFINITION OF OPTION BENEFITS. Options provide benefit to research by both directly and indirectly increasing the three research metrics: Research Diversity, Number of Research Facilities Operable, and the Hours of Research. All three metrics are based on a 90 day Research Quarter (RQ). Research Facilities Operable and Hours of Research are outputs of the Monte Carlo-based statistical model employed by the program, called the Payload Utilization Modeler (PLUM) tool. The PLUM operator calculates Research Diversity separately using information provided by the PLUM tool. These three metrics form the basis of the TREDS tool input. Option benefits should not be confused with option resources, which are the raw capabilities provided/required 
by the options. Examples of resources include space shuttle mid-deck locker space and electrical power required.

D) DEFINITION OF OPTION COSTS. In every study, both developmental and operational costs were obtained for each option. It is important to include both categories, as the composition of option costs can range from entirely operational to almost entirely developmental. To facilitate the gathering of cost information and provide consistency in the financial data reported, a representative from the program business management office was identified. All costs used in the studies reflect an escalation factor to account for inflation.

E ) DEFINITION OF OPTION RISKS. Risk assessment was performed by the study team and certified by the program risk manager. A modified version of the ISS Program risk matrix is used to disclose the probability and consequence scores for risks assessed for each option. Modifications consisted of establishing likelihood and consequence ranges and thresholds for risks affecting the implementation of the research enhancement options and their impact to the research enhancement potential. This facilitates mathematical inclusion of identified risks in the calculation of research return. Risks are assessed in four categories:

- Technical implementation risks

- Schedule implementation risks

- Cost risks

- Increased safety risk caused by the implementation of each option.

The TREDS tool can accept either likelihood scores directly, as percentages, in addition to accepting them in the form of levels 1 through 5 . This was done to accommodate risk likelihoods as they are produced by the ISS program's Probabilistic Risk Assessment (PRA) capability.

F) PERFORMANCE OF ANALYSES. Once data collection was complete, the TREDS runs were performed. The use of TREDS is straightforward since the tool is based on Microsoft Excel. Results of the TREDS outputs are summarized below.

The primary outputs of TREDS are charts showing the cumulative return of the options over the study timeframe (relative to baseline) versus their cost. Two variations of these charts are available; one shows option costs in absolute dollars, while the other shows option costs as a percentage of total ISS program costs (Figure 3). Risk penalties are assessed against the projected benefit and cost for the options. The benefit penalty is assessed based on the level of technical and schedule risks that were determined and 
scored for each option. These penalties are discounted from the projected benefit using specialized algorithms developed for this purpose and are represented in the figure as a vertical line extending downward from the cost benefit point. The cost risk for each option is assessed as a percentage range around the cost estimate and is represented as a horizontal line crossing the cost benefit point.

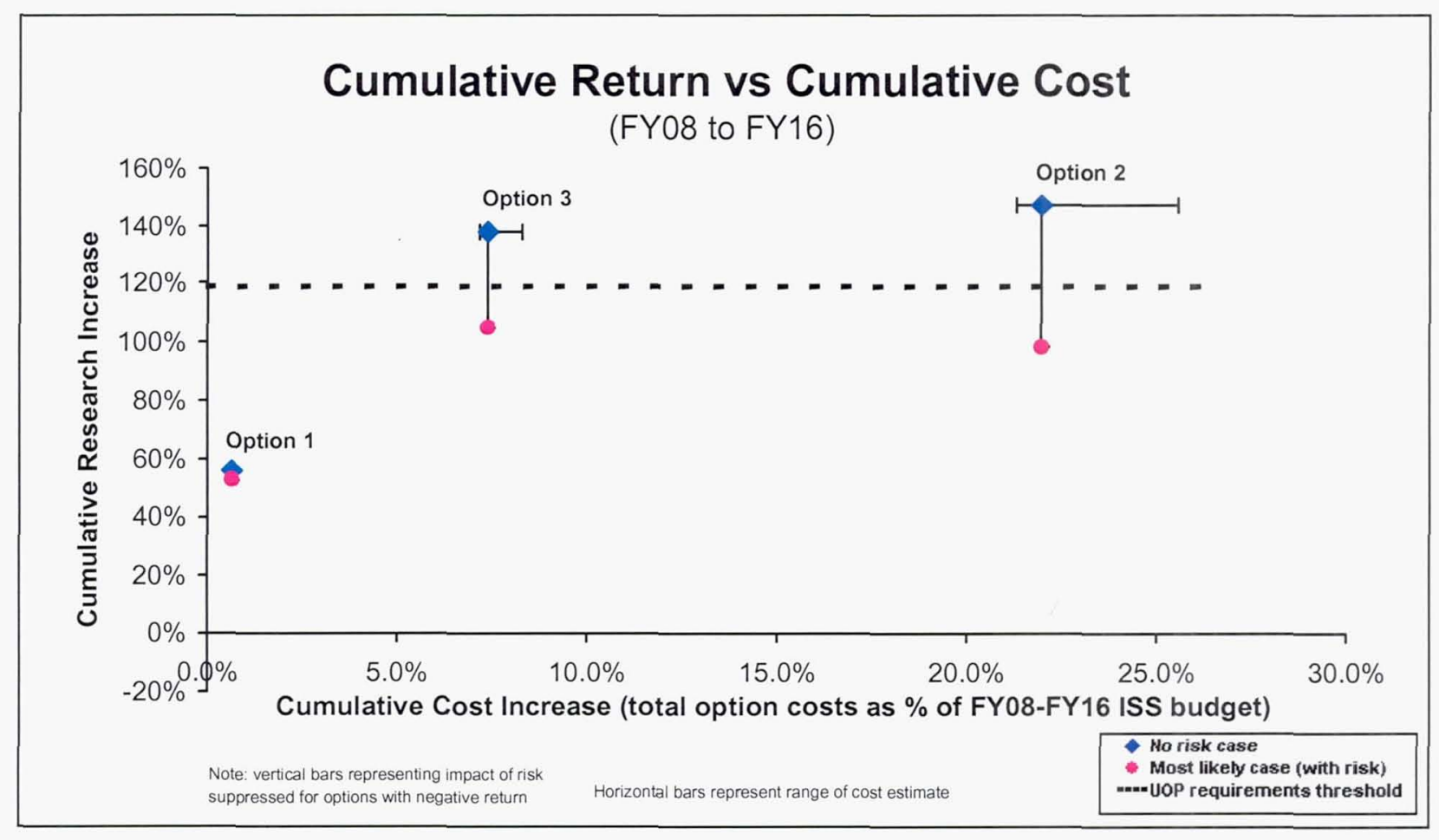

Figure 3. TREDS Summary Output

The option with the most favorable benefit to cost ratio can be identified by superimposing a line over the Y-axis and rotating it in a clockwise direction. The first option crossed by the rotating line is the most favorable. Using this technique on Figure 3 identifies Option 1 as having the most favorable benefit to cost ratio. Option 1 also shows relatively little performance and schedule risk penalty. Option 3 is a distant second, and Option 3 offers little more benefit than Option 2 at a substantially higher cost. Both Option 2 and 3 have the potential for substantial performance and schedule risk penalties as well as a greater cost risk. These two options do, however, offer benefit in excess of program requirements. Option 1 falls well short of these requirements.

A second output is the research increase vs. time chart, shown in Figure 4. This output was created to graphically demonstrate the phasing of option returns. In the example shown in Figure 4, two baselines are included ("US Core" and "IP Core") and the start dates of the options varied. It should be noted that in Figure 4, increases in research return provided by the options are shown as a research multiple, i.e. the net increase in 
research capability provided by the option at a given time interval (as opposed to the cumulative returns shown in Figure 3). This information is very useful in determining the optimal time to phase-in or to discontinue a given option. For example, Option 1 in Figure 4 provides research capability enhancement of around $90 \%$ as compared to the baseline during 2005-2007 time frame. After 2007, the baseline configuration changes and this option will now provide a lesser return of about $50 \%$ over the baseline. The decision maker will have to reconsider the total benefit-cost-risk case for this option after 2007 to determine if implementation is still beneficial to the program.

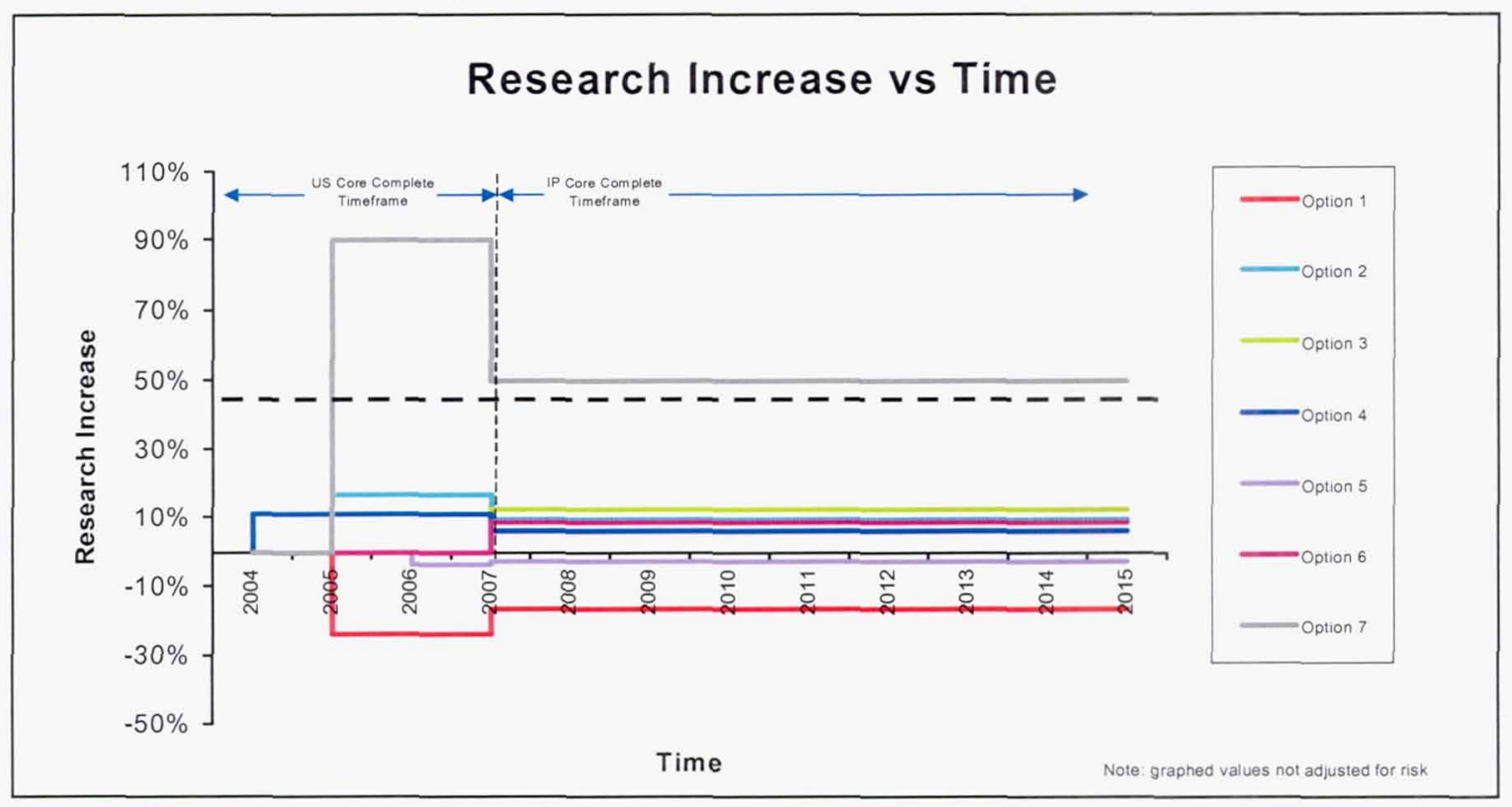

Figure 4. TREDS Research Multiple vs. Time

Figure 5 shows another output of TREDS that is used to compare the relative level of risks between the options. An important component of this graphic is the representation of the relative level of safety risk inherent between options. This is an important element, for obvious reasons, in discriminating between alternative options. This graphic provides another dimension for the decision maker in terms of risk since the risk information provided in Figure 3 is mainly based on assessing penalties on projected research enhancement potential.

Other graphical outputs are also available such as the cost breakdown by fiscal year shown to allow a side-by-side comparison of the costs of each option. Cost spikes, timing of costs and cost escalation over time are readily apparent. This information is important for comparison and reconciliation with the available funding profile for the Program. 


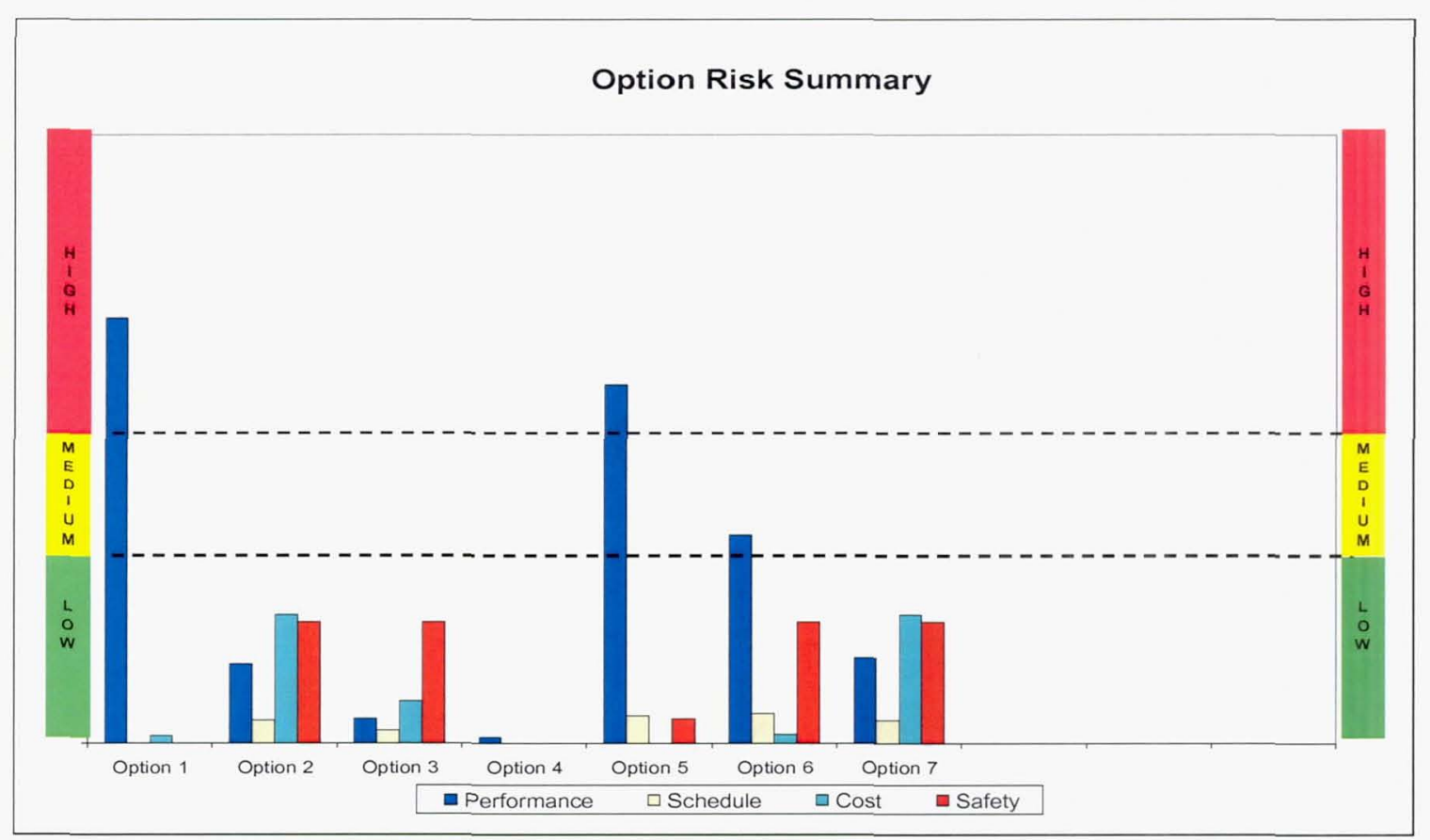

Figure 5. TREDS options risk summary

\section{Observations}

After the conduct of two full studies to verify and validate the framework, the study team found that the methodology and TREDS add value to ISS program decisionmaking. Specifically, the methodology:

- Provides a disciplined approach.

- Lends consistency to the evaluation of different options.

- Brings together the fundamental decision components: cost, benefit, and risk.

- Is mathematical, repeatable, and comprehensive; detailed quantitative approaches are infused at an elementary level and documented.

- Provides a uniform, visible, and documented framework for decision advocacy both inside and outside the Program.

- Improves communication and integration among the different disciplines necessary to the studies process: business management, performance assessment teams, risk management, research planning, operations \& integration, and technical study leads.

- Allows manipulation to examine sensitivities and investigate what-if scenarios. 
- Allows comparisons and helps focus on what should be studied in detail.

The methodology does, however, have certain limitations:

- It does not give a final answer in all cases. Option optimization will still be necessary to obtain the complete picture. Unknowns or poor definitions can drive the assumptions, and results can vary significantly based on those assumptions and on the fidelity of data. The Program Manager should use the results provided by the TREDS tool as an input to decision making.

- Implementation of the methodology requires an investment. The increased level of rigor in the definition and analysis of the alternatives may require additional expenditure of resources such as manpower and computer time.

\section{Conclusion}

Prior to development and operational use of this framework, the ISS Program Manager was provided limited and dissimilar information in which to base programmatic decisions concerning the utilization potential of the ISS. Limitations included the need to develop a quantitative measure of research potential to perform cost benefit trades, the need to adjust the assessed benefit based on implementations risks, and the need to improve the systematic assessment of costs. The framework described in this paper addressed all of these limitations and provided an integrated system to perform feasibility studies to evaluate and rank research enhancement options. This framework is a fine example of systems engineering practice to improve the execution of a complex operational program.

\section{References}

Saaty, T.L., "Priority Setting in Complex Problems." IEEE Transactions in Engineering Management," Vol. EM-30, NO. 3, August 1983 